\title{
Circular RNA ATAD1 is upregulated in acute myeloid leukemia and promotes cancer cell proliferation by downregulating miR-34b via promoter methylation
}

\author{
YARONG WU ${ }^{1}$, BINGJUN GAO ${ }^{2}$, XIAOLEI QI ${ }^{3}$, LIYUN BAI $^{3}$, BIXIN $^{3} I^{3}$, \\ HONGJING BAO ${ }^{4}, \mathrm{XI} \mathrm{WU}^{5},{\mathrm{XIAOYUN} W \mathrm{~W}^{6} \text { and YUXIAZHAO }}^{3}$
}

\begin{abstract}
Departments of ${ }^{1}$ Hematology and ${ }^{2}$ Osteology, The People's Hospital of Danyang, Affiliated Danyang Hospital of Nantong University, Danyang, Jiangsu 212300; Departments of ${ }^{3}$ Hematology, ${ }^{4}$ Ultrasound and ${ }^{5}$ Neurosurgery,

The People's Hospital of Xing'an League, Ulanhot, Inner Mongolia Autonomous Region 137499;

${ }^{6}$ Department of Technology, Research Center for Hua-Da Precision Medicine of Inner Mongolia

Autonomous Region, Hohhot, Inner Mongolia Autonomous Region 010000, P.R. China
\end{abstract}

Received November 21, 2020; Accepted July 16, 2021

DOI: 10.3892/ol.2021.13060

\begin{abstract}
A previous study has reported the oncogenic role of circular RNA (circ)-ATAD1 in gastric cancer. The aim of the present study was to investigate the role of circ-ATAD1 in acute myeloid leukemia (AML). Bone marrow mononuclear cells were collected from 60 patients with AML and 60 healthy controls, followed by RNA isolation and reverse transcription-quantitative PCR to assess the expression of circ-ATAD1 and microRNA (miR)-34b. A subcellular fractionation assay was used to determine the subcellular location of circ-ATAD1 in AML cells. Furthermore, circ-ATAD1 and miR-34b were overexpressed in AML cells to study crosstalk between the two molecules. The effect of circ-ATAD1 overexpression on miR-34b gene methylation was also analyzed by methylation-specific PCR, and the roles of circ-ATAD1 and miR-34b in the regulation of AML cell proliferation were analyzed by BrdU assay. circ-ATAD1 expression was found to be elevated, and inversely correlated with that of miR-34b, in patients with AML. Subcellular fractionation assays showed that circ-ATAD1 was specifically expressed in the nucleus. In addition, circ-ATAD1 overexpression in AML cells decreased miR-34b expression and increased miR-34b gene
\end{abstract}

Correspondence to: Dr Yuxia Zhao, Department of Hematology, The People's Hospital of Xing'an League, 66 Hanshan West Street, Ulanhot, Inner Mongolia Autonomous Region 137499, P.R. China E-mail: YuxiaZhaoInner@163.com

Abbreviations: AML, acute myelogenous leukemia; BMMNCs, bone marrow mononuclear cells; FISH, fluorescence in situ hybridization; IF, immunofluorescence; MSP, methylation-specific PCR; USP, unmethylation-specific PCR; LICs, leukemia initiating cells

Key words: acute myeloid leukemia, circular RNA ATAD1, microRNA-34b, proliferation, methylation methylation. Moreover, AML cell proliferation was increased by circ-ATAD1 overexpression, but decreased by miR-34b overexpression, and the effect of circ-ATAD1 overexpression on AML cell proliferation was reduced by miR-34b overexpression. Together, these results indicate circ-ATAD1 as a nucleus-specific circRNA in AML, which promotes AML cell proliferation by downregulating miR-34b via methylation.

\section{Introduction}

Acute myelogenous leukemia (AML) is the most common type of acute leukemia, accounting for $\sim 80 \%$ of all cases worldwide (1). AML develops from the bone marrow and blood, and is characterized by rapid progression and a highly aggressive nature (2). Although AML is a severe malignancy, it is treatable and often curable with chemotherapy in combination with targeted drugs $(3,4)$. However, chemoresistance frequently occurs after long-term therapy, resulting in chemotherapeutic failure and poor patient survival times $(5,6)$. Therefore, the development of novel therapeutic approaches to further improve patient survival is urgently required.

Molecular-targeted therapies are emerging novel approaches for AML treatment, which regulate the expression of related genes (7-9). With an increasing understanding of the molecular mechanisms of AML, certain molecular pathways, such as PI3K/Akt/mTOR signaling and glutathione metabolism pathways, have been indicated as potential targets for anti-AML therapy $(10,11)$. However, molecular-targeted therapy is still in the research stages. Assessment of therapeutic safety, and the identification of more effective drug targets, are being widely researched. As covalently closed, single-strand RNA transcripts, circular RNAs (circRNAs) are involved in various cancer types by regulating the expression of cancer-related genes (12). Noncoding RNAs have been regarded as novel regulators of cancer progression (13). microRNAs (miRNAs/miRs) and circRNAs are two important types of noncoding RNA. MiR-34b is one of the hallmark miRNAs that are associated with AML chemotherapy resistance (14). Furthermore, 
miR-34b regulates ubiquitin-specific protease $2 \mathrm{a}$ expression to increase intracellular glutathione content and indirectly interfere with the oxidative cascade, triggered by chemotherapeutic agents (15). Circ-ATAD1 is a newly identified circRNA contributing to gastric cancer cell progression (16). However, to the best of our knowledge, its role in other cancer types is yet to be reported. Therefore, the present study was conducted to investigate the role of circ-ATAD1 and miR-34b in AML.

\section{Materials and methods}

Research subjects. A total of 60 patients with AML (38 men and 22 women; age, 62.2+/-5.7 years) and 60 healthy controls (38 men and 22 women; age, 62.1+/-5.8 years) who were admitted to the Xing'an League People's Hospital (Ulanhot, China) between May 2017 and May 2020, were enrolled in the present study. All healthy controls showed normal physiological parameters in systemic physiological examination, including bone marrow blasts $<4 \%$, and had no history of AML. All patients were diagnosed using a bone marrow test. All enrolled patients had a percentage of bone marrow blasts $>20 \%$ (with a mean of $21.5 \%$ ) and no previous history of AML or other malignancies. Patients with other clinical disorders (such as metabolic disorders, chronic diseases and severe infection), and who had initiated therapy for such disorders within 3 months prior to admission, were excluded. The present study was approved by the Ethics Committee of Xing'an League People's Hospital (approval no. 323SE), and all patients and control subjects provided written informed consent.

Bone marrow mononuclear cells (BMMNCs) and AML cell lines. Bone marrow was collected from all patients and healthy controls by biopsy, and used to isolate BMMNCs using lymphocyte separation medium (TBD Science; Tian Jin Hao Yang Biological Manufacture Co., Ltd.). The isolation procedure was conducted following the manufacturer's instructions. Briefly, $2 \mathrm{ml}$ bone marrow mixed with medium was centrifuged at $400 \mathrm{x} \mathrm{g}$ for $15 \mathrm{~min}$. The second layer of the supernatant (containing the lymphocytes) was then used for cell culture. The Beckman MoFlo Astrios high-performance, live-cell sorting system (Beckman Coulter, Inc.) was used to isolate BMMNC subpopulations, as outlined in a previous study (17). PE-conjugated mouse anti-human CXCR4 (cat. no. 60089PE.1; Stemcell Technologies, Inc.) and APC-conjugated rat anti-human CD45 (cat. no. 28145-1; Signalway Antibody LLC) were used to isolate the BMMNCs, per the sorting identification strategy of a previous study (17). The cells were resuspended in minimal essential medium with Earle's salts (Gibco; Thermo Fisher Scientific, Inc.) containing $10 \%$ fetal calf serum and $1 \%$ antibiotics (penicillin/streptomycin; Gibco; Thermo Fisher Scientific, Inc.); $1 \times 10^{7}$ resuspended bone marrow cells were seeded into $100-\mathrm{mm}$ cell culture dishes and incubated at $37^{\circ} \mathrm{C}$ in a humidified incubator with 5\% $\mathrm{CO}_{2}$. Kasumi-3 and Kasumi-6 AML cell lines were purchased from the ATCC, and cultured in RPMI-1640 medium (10\% FBS) at $37^{\circ} \mathrm{C}$ in an incubator with $5 \% \mathrm{CO}_{2}$ and $95 \%$ humidity. Cells used in the subsequent assays were collected at $\sim 85 \%$ confluence.

Transient transfection. Overexpression of circ-ATAD1 and miR-34b was achieved by transfecting $1 \times 10^{6}$ Kasumi-3 or
Kasumi-6 cells with a circ-ATAD1 expression vector $(1 \mu \mathrm{g}$; pcDNA3.1 backbone vector; Invitrogen; Thermo Fisher Scientific, Inc.) or miR-34b mimics (10 nM; Sigma-Aldrich; Merck KGaA) using Lipofectamine ${ }^{\circledR} 2000$ (Invitrogen; Thermo Fisher Scientific, Inc.) (1:2). Untransfected and empty vector- or NC miRNA-(Sigma-Aldrich; Merck KGaA) transfected cells were used as the normal control and negative control (NC) cells, respectively. The sequences of the miR-34b mimics and miR-NC are displayed in the Table I. After incubation with the transfection mixture for $6 \mathrm{~h}$ at $37^{\circ} \mathrm{C}$, cells were immediately washed with fresh medium, followed by culture in fresh medium for another $48 \mathrm{~h}$ prior to use.

$R N A$ isolation and reverse transcription-quantitative (RT-q) $P C R$. Total RNA was isolated from BMMNCs and in vitro cultured cells using RNAzol reagent (Sigma-Aldrich; Merck $\mathrm{KGaA}$ ), and treated with DNA eraser (Takara Bio, Inc.) at $37^{\circ} \mathrm{C}$ until the OD260 $\mathrm{nm} / 280 \mathrm{~nm}$ ratio had reached $\sim 2.0$ (pure RNA). The integrity of all RNA samples was determined by electrophoresis using a 5\% urea-PAGE gel. Total RNA was used for first-strand cDNA synthesis with the Prime Script RT reagent kit (Takara Bio, Inc.) per the manufacturer's instructions. Circ-ATAD1 expression was determined using the SYBR ${ }^{\circledR}$ Green Quantitative RT-qPCR Kit (Sigma-Aldrich; Merck KGaA) with GAPDH as the internal control. Mature miR-34b expression was analyzed using the All-in-One ${ }^{\mathrm{TM}}$ miRNA qRT-PCR Detection Kit (GeneCopoeia, Inc.) with U6 as the internal control. All operations were completed following the manufacturers' instructions. The qPCR thermocycling conditions for all genes, cirRNA and miRNAs were as follows: $95^{\circ} \mathrm{C}$ for $1 \mathrm{~min}$, followed by 40 cycles of $95^{\circ} \mathrm{C}$ for $10 \mathrm{sec}$ and $60^{\circ} \mathrm{C}$ for $45 \mathrm{sec}$. PCR reactions were performed using the CFX96 Touch Real-Time PCR Detection System (Bio-Rad Laboratories, Inc.). Ct values of the targeted genes were normalized to the corresponding internal controls based on the $2^{-\Delta \Delta \mathrm{Cq}}$ method, which was used to quantify gene expression (18). The qPCR primers are listed in Table II.

Subcellular fractionation assay. Both the nuclear and cytoplasmic fractions of Kasumi-3 and Kasumi-6 cells were prepared using the Nuclei Isolation Kit: Nuclei EZ Prep (Sigma-Aldrich; Merck KGaA) according to the manufacturer's instructions, and used for RNA isolation and RT-qPCR to detect circ-ATAD1, with GAPDH as the internal control.

Fluorescence in situ hybridization (FISH) and immunofluorescence (IF). IF staining with anti-histone H3 (1:300; cat. no. ab6002; Abcam) was in reference to a previous study (19). The Blocking buffer was PBS with $1 \%$ BSA (Thermo Fisher Scientific, Inc.). The Alexa fluor 555 anti-rabbit antibody (cat. no. ab150078; Abcam) was used as the secondary antibody (1:500). FISH was performed using Kasumi-3 and Kasumi-6 cells as described previously (19). The probe of cir-ATAD1 (TACCACAGCCTGGAGGCC CATAG) was synthesized and labeled with digoxigenin (DIG-dUTP) by Sangon Biotech (Shanghai) Co., Ltd. Specifically, the slices covered by cells were fixed in $4 \%$ paraformaldehyde (MilliporeSigma) for $10 \mathrm{~min}$ at room temperature. Before pre-hybridization, cells were permeabilized with cold $0.1 \%$ Triton X-100 and pre-hybridized with 
Table I. Sequences of oligonucleotides.

Oligonucleotide

Sequence $\left(5^{\prime}-3^{\prime}\right)$

Mimics NC sense

Mimics NC antisense

UUCUCCGAACGUGUCACGUU

miR-34b mimics sense

AACGUGACACGUUCGGAGAA

miR-34b mimics antisense

UAGGCAGUGUCAUUAGCUGAUUG

CAAUCAGCUAAUGACACUGCCUA

NC, negative control; miR, microRNA.

Table II. Sequences of quantitative PCR primers.

\begin{tabular}{lll}
\hline Gene & \multicolumn{1}{c}{ Forward primer $\left(5^{\prime}-3^{\prime}\right)$} & \multicolumn{1}{c}{ Reverse primer $\left(5^{\prime}-3^{\prime}\right)$} \\
\hline Circ-ATAD1 & GTTTCCTTCCTGTGTGAGGC & GGTCCGAGACGGTCCTTAAA \\
U6 & CTCGCTTCGGCAGCACA & AACGCTTCACGAATTTGCGT \\
miR-34b & AGGCAGTGTCATTAGCTGATTGT & ACAATCAGCTAATGACACTGCCT \\
GAPDH & CCATTTGCAGTGGCAAAG & CACCCTTTGTGTTAGTG \\
\hline
\end{tabular}

Table III. Sequences of primers used for MSP.

\begin{tabular}{lll}
\hline Gene & \multicolumn{1}{c}{ Forward primer $\left(5^{\prime}-3^{\prime}\right)$} & Reverse primer $\left(5^{\prime}-3^{\prime}\right)$ \\
\hline miR-34b USP & TTTTTATTTGTTTTGTTTTGTGTTTGTTTTG & CAACTACAACTCCCAAACAATCC \\
miR-34b MSP & ATTCGTTTCGTTTCGCGTTCGTTTC & CGACTACAACTCCCGAACGATCCG
\end{tabular}

MSP, methylation-specific PCR. USP, Unmethylation-specific PCR.

a hybridization buffer at $37^{\circ} \mathrm{C}$ for $1 \mathrm{~h}$. The slides were incubated with a hybridization buffer containing the FISH probe at $95^{\circ} \mathrm{C}$ for $5 \mathrm{~min}$, and then at $37^{\circ} \mathrm{C}$ overnight in the dark in a humid chamber. The samples were washed with $2 \times$ saline sodium citrate buffer (SSC) for $10 \mathrm{~min}$ at $37^{\circ} \mathrm{C}, 1 \times \mathrm{SSC}$ for $2 \times 5 \mathrm{~min}$ at $37^{\circ} \mathrm{C}$, and $0.5 \times \mathrm{SSC}$ for $10 \mathrm{~min}$ at room temperature. The slides were then incubated with anti-DIG-488 (1:300; cat. no. ab150077; Abcam) at $37^{\circ} \mathrm{C}$ for $50 \mathrm{~min}$, and the nuclei were counterstained with DAPI at room temperature for $30 \mathrm{~min}$. Finally, the slices were sealed in fluorescence decay-resistant medium and images were obtained under a fluorescence microscope (Nikon Corporation).

Methylation-specific PCR (MSP). Genomic DNA isolation from transfected Kasumi-3 and Kasumi-6 cells was performed using a routine method (20). All genomic DNA samples were processed using the EZ DNA Methylation-Gold ${ }^{\mathrm{TM}}$ Kit (Zymo Research Corp.) per the manufacturer's protocol. Then, qPCR and routine PCR were performed to detect methylation of the miR-34b gene promoter using PCR Master Mix x2 (Invitrogen; Thermo Fisher Scientific, Inc.). The MSP primers can be used to amplify methylated template, while the primers for unmethylation-specific PCR (USP) do not amplify these products. Both the MSP and USP conditions were as following: $95^{\circ} \mathrm{C}$ for $5 \mathrm{~min}$, followed by 40 cycles of $95^{\circ} \mathrm{C}$ for $30 \mathrm{sec}, 55^{\circ} \mathrm{C}$ for $30 \mathrm{sec}$ and $72^{\circ} \mathrm{C}$ for $50 \mathrm{sec}$, and then $72^{\circ} \mathrm{C}$ for $10 \mathrm{~min}$. All primers used for MSP and USP are listed in Table III.

BrdU assay. A total of $3 \times 10^{3}$ transfected Kasumi-3 and Kasumi-6 cells were transferred to each well of a 96-well plate in $0.1 \mathrm{ml}$ medium, and cultured at $37^{\circ} \mathrm{C}$ for $48 \mathrm{~h}$ before the addition of BrdU. The experiment was conducted using the BrdU Cell Proliferation Assay (cat. no. QIA58; Sigma-Aldrich; Merck KGaA) according to the manufacturer's protocol. Then, cells were cultured with $20 \mu \mathrm{l} /$ well diluted BrdU reagent $(10 \mathrm{mM})$ for $6 \mathrm{~h}$, fixed with Fixing solution and incubated for $30 \mathrm{~min}$. After fixation, the cells were incubated for another $48 \mathrm{~h}$ with peroxidase-coupled anti-BrdU-antibody (supplied by the kit), followed by washing twice with ice-cold PBS. After incubation with peroxidase substrate for $3 \mathrm{~h}$, OD values were measured at $450 \mathrm{~nm}$. For the represent images, the anti-Brdu (1:500; cat. no. ab6362; Abcam) was used as primary antibody, and Goat anti-rabbit Alexa Fluor ${ }^{\circledR} 546$ (1:2,000; cat. no. A11010; Invitrogen; Therno Fisher4 Scientific, Inc.) was used as the secondary antibody. The detailed method has been previously published (21).

Statistical analysis. AML and control groups were compared by unpaired t-test. Comparisons among multiple independent 
A

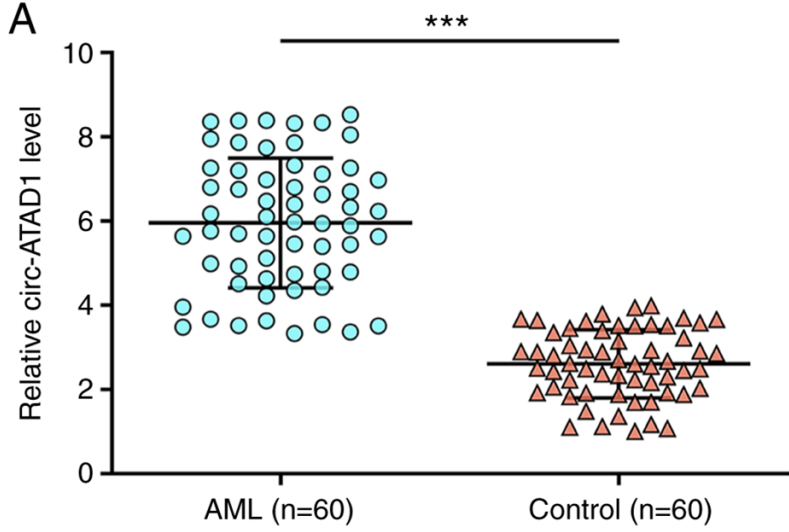

C

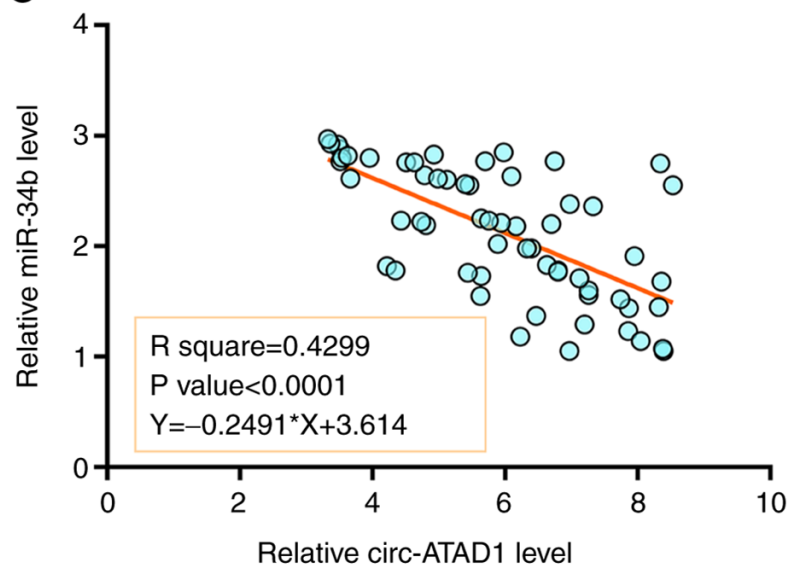

B

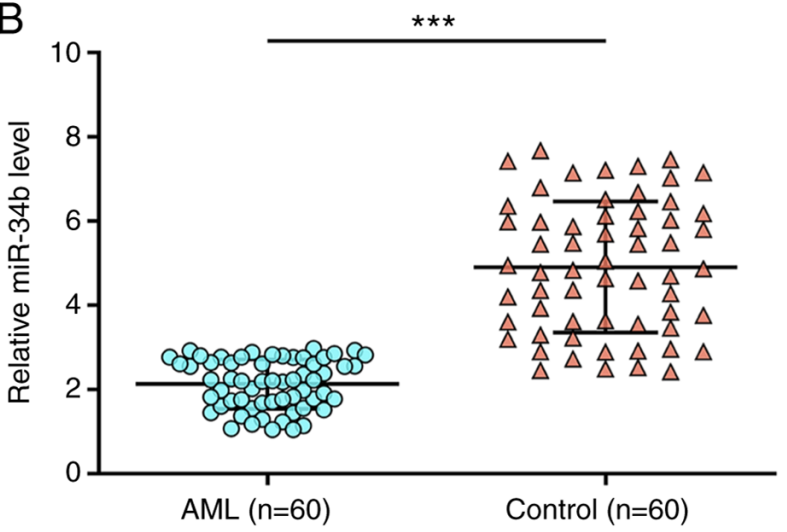

$\mathrm{D}$

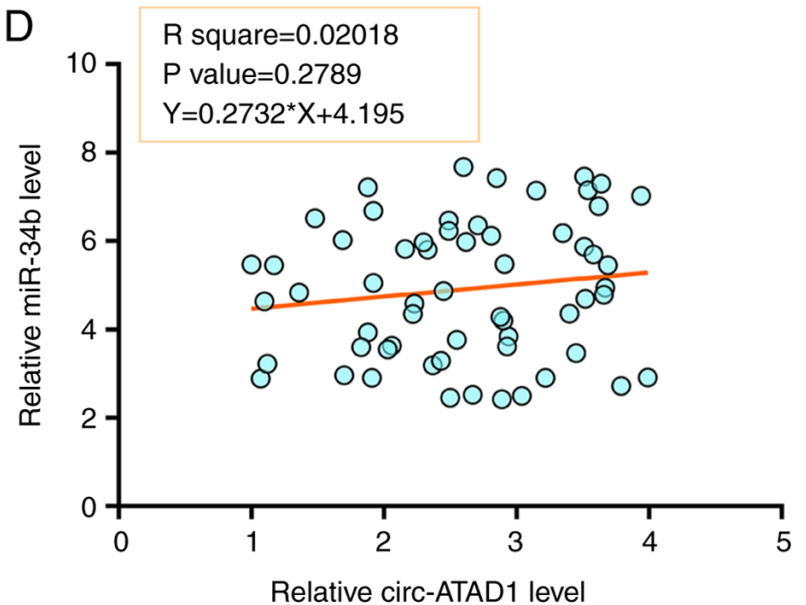

Figure 1. Expression of circ-ATAD1 and miR-34b is altered in patients with AML. Samples of bone marrow mononuclear cells from patients with AML $(n=60)$ and healthy controls $(n=60)$ were subjected to RNA isolation and reverse transcription-quantitative PCR to determine the differential expression of (A) circ-ATAD1 and (B) miR-34b. To study the crosstalk between circ-ATAD1 and miR-34b, correlation analyses across both (C) AML and (D) control samples were conducted by Pearson's correlation coefficient. ${ }^{* * *} \mathrm{P}<0.01$. circ, circular RNA; AML, acute myelogenous leukemia; miR, microRNA.

cell transfection groups were analyzed by one-way ANOVA followed by Tukey's test. Correlations were analyzed by Pearson's correlation coefficient. Data are presented as the mean \pm standard deviation of three independent replicates. $\mathrm{P}<0.05$ was considered to indicate a statistically significant difference.

\section{Results}

Patients with AML exhibit altered expression of circ-ATADI and miR-34b. Samples of BMMNCs from both patients with AML $(n=60)$ and healthy controls $(n=60)$ were subjected to RNA isolation and RT-qPCR to determine the differential expression of circ-ATAD1 and miR-34b. Compared with the controls, circ-ATAD1 was highly expressed in AML (Fig. 1A, $\mathrm{P}<0.01$ ), while miR-34b expression was lower in AML (Fig. 1B, P<0.01), suggesting that circ-ATAD1 upregulation and miR-34a downregulation may be involved in AML. To study the crosstalk between circ-ATAD1 and miR-34b, their correlations across both AML and control samples were determined using Pearson's correlation analysis. The data showed that circ-ATAD1 and miR-34b were closely and inversely correlated across AML samples (Fig. 1C; $\mathrm{P}<0.0001$ ), but not across the control samples (Fig. 1D). Therefore, circ-ATAD1 and miR-34b may interact with each other in AML.
circ-ATAD1 overexpression decreases $m i R-34 b$ expression in AML cells. To further study the crosstalk between circ-ATAD1 and miR-34b, Kasumi-3 and Kasumi- 6 cells were transfected with either a circ-ATAD1 overexpression vector or miR-34b mimics, followed by expression confirmation every $24 \mathrm{~h}$ until $96 \mathrm{~h}$. It was observed that both circ-ATAD1 and miR-34b were overexpressed between 24 and 96 h post transfection (Fig. 2A and $\mathrm{B}, \mathrm{P}<0.05$ ). In addition, circ-ATAD1 overexpression decreased that of miR-34b (Fig. 2C, P<0.05). By contrast, miR-34b overexpression failed to significantly alter circ-ATAD1 expression (Fig. 2D). Therefore, circ-ATAD1 may downregulate miR-34b expression in AML cells.

Circ-ATAD1 is a nucleus-specific circRNA that increases miR-34b gene methylation in AML cells. A subcellular fractionation assay was used to determine the subcellular location of circ-ATAD1 in both Kasumi-3 and Kasumi-6 cells, and circ-ATAD1 was only detected in the nuclear fractions (Fig. 3A). The relative expression of circ-ATAD1 and miR-34b in Kasumi-3 cells (nuclei), in Kasumi-6 cells (nuclei), in the control and that in the AML patient samples is also provided. The expression levels of circ-ATAD1 were highest in AML samples (Fig. 3B; $\mathrm{P}<0.01$ ), and those of miR-34b were highest in the healthy control samples (Fig. 3C; P<0.01). FISH (Figs. S1 and S2) also showed 
A

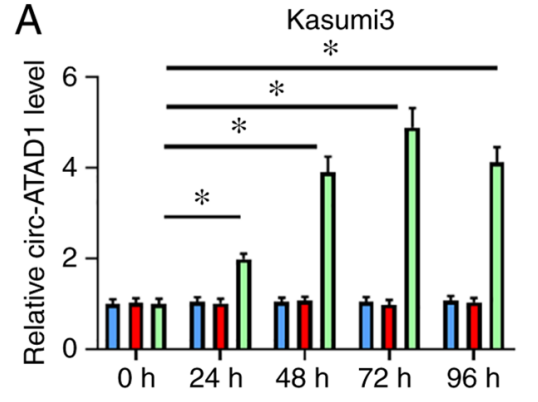

B
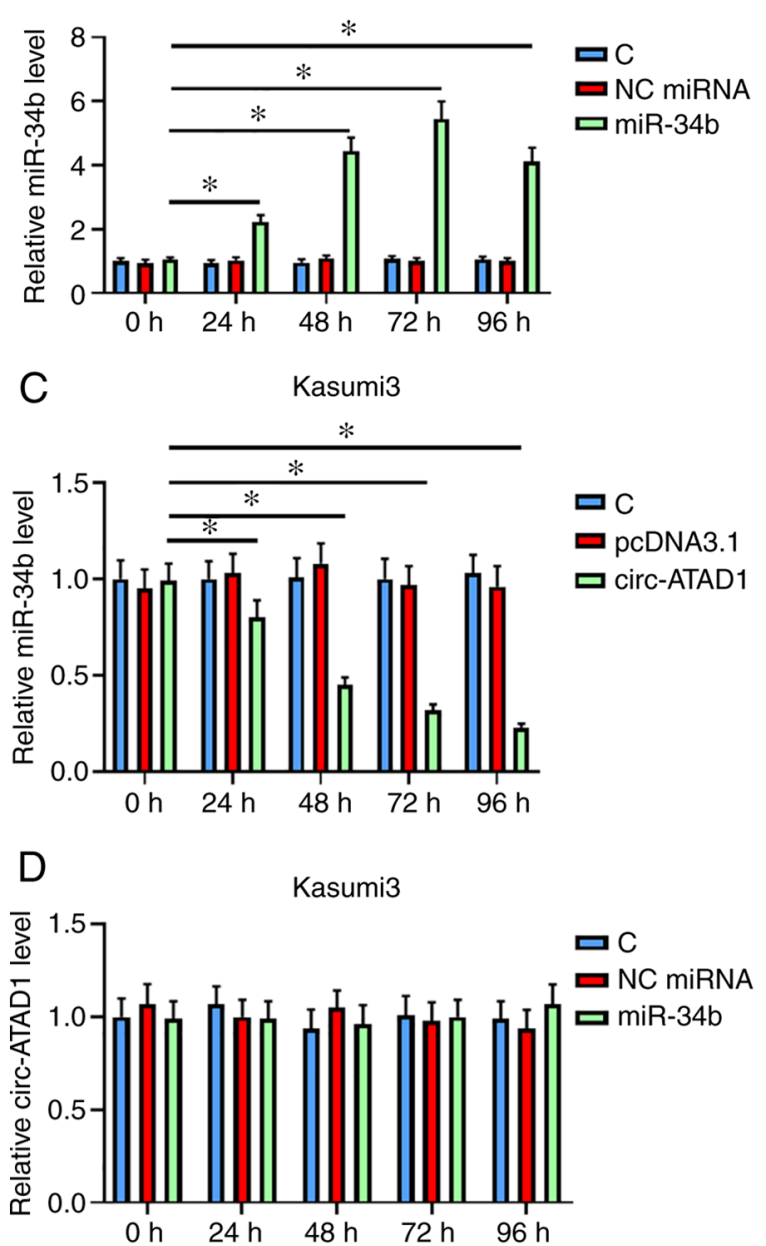

Kasumi6
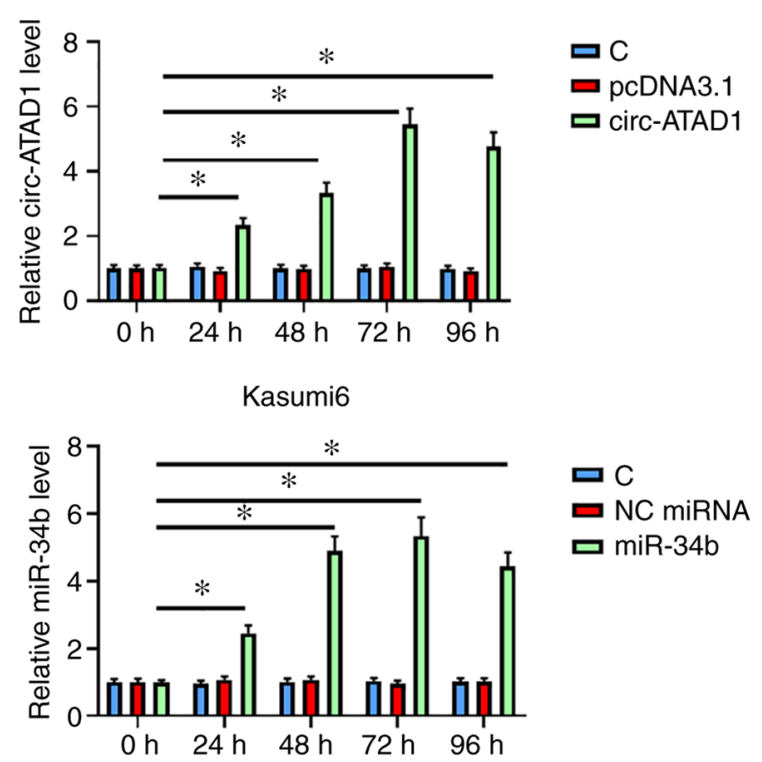

Kasumi6
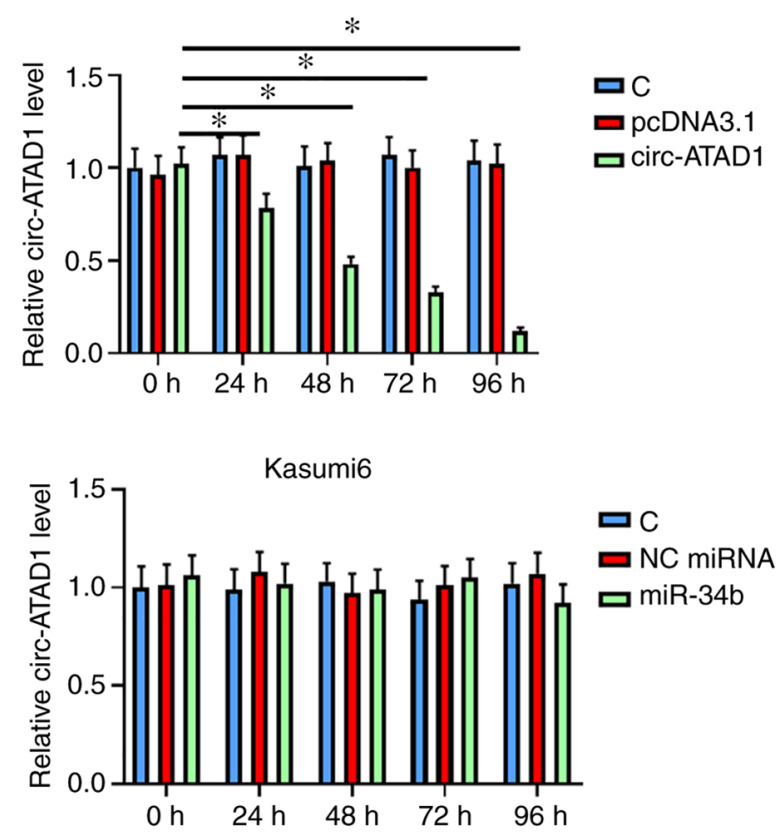

Figure 2. Circ-ATAD1 overexpression decreases miR-34b expression in AML cells. (A) Kasumi-3 and (B) Kasumi-6 cells were transfected with either circ-ATAD1 expression vector or the miR-34b mimics, followed by confirmation of overexpression every $24 \mathrm{~h}$ until $96 \mathrm{~h}$. Relative expression of (A) circ-ATAD1 and (B) miR-34b in Kasumi-3 and Kasumi-6 cells were compared between 24 and 96 by RT-qPCR. Effects of (C) circ-ATAD1 overexpression on miR-34b expression, and the effects of (D) miR-34b overexpression on circ-ATAD1 expression were also analyzed by RT-qPCR. Data are presented as the mean \pm SD of three independent replicates. ${ }^{*} \mathrm{P}<0.05$. circ, circular RNA; miR, microRNA; AML, acute myelogenous leukemia; C, control; NC, negative control; RT-q, reverse transcription-quantitative.

similar results. In addition, the effects of circ-ATAD1 overexpression on miR-34b gene methylation were analyzed by MSP. Compared with cells transfected with empty pcDNA3.1 vector, cells transfected with the circ-ATAD1 expression vector showed increased methylation of miR34b (Fig. 3D). These results indicate the significantly different expression of these two molecules in healthy and tumor cells. In addition, the expression of miR-34b was regulated by circ-ATAD1 through methylation-related mechanisms in Kasumi-3 and Kasumi-6 cells.
Circ-ATAD1 overexpression promotes the proliferation of $A M L$ cells through miR-34b. The role of circ-ATAD1 and miR-34b in regulating Kasumi-3 (Fig. 4A and C) and Kasumi-6 (Fig. 4B and D) cell proliferation was analyzed using a BrdU assay. Circ-ATAD1 overexpression increased cellular proliferation, while miR-34b overexpression decreased cellular proliferation (both $(\mathrm{P}<0.05)$. Moreover, co-transfection analysis showed that the effect of circ-ATAD1 overexpression on AML cell proliferation was reduced by miR-34b overexpression $(\mathrm{P}<0.05)$. The results indicated Circ-ATAD1 overexpression 

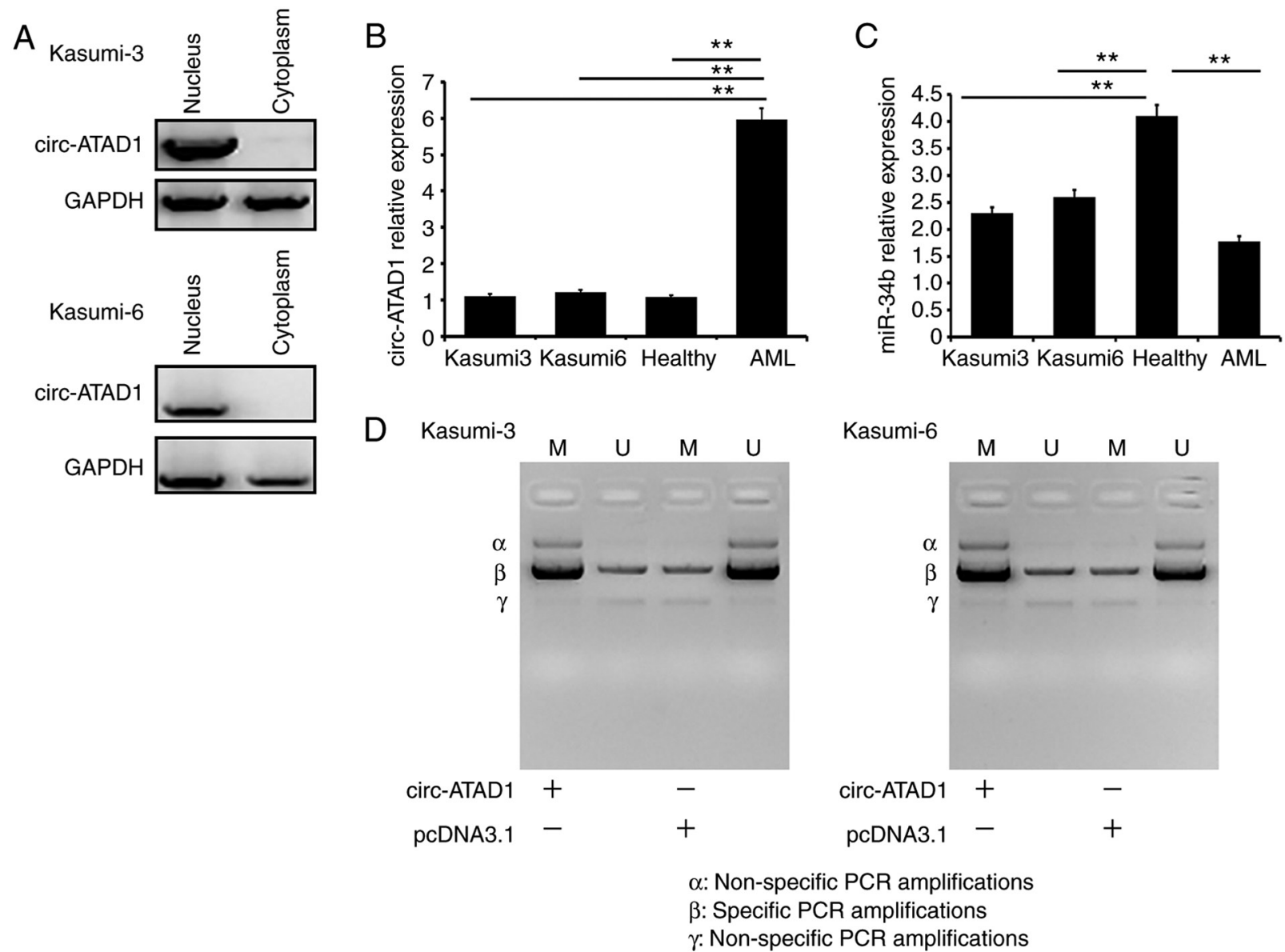

Figure 3. Circ-ATAD1 is a nucleus-specific circRNA that increases methylation of the miR-34b gene in AML cells. (A) Subcellular fractionation assays were used to determine the subcellular location of circ-ATAD1 in both Kasumi-3 and Kasumi- 6 cells. Relative expression of (B) circ-ATAD1 and (C) miR-34b in Kasumi-3 cells (nuclei), Kasumi-6 (nuclei) cells and (C) samples from healthy control and patients with AML. Expression of GAPDH or U6 in the nuclei of Kasumi-3 and Kasumi- 6 cells was normalized to ' 1 '. Effects of circ-ATAD1 overexpression on miR-34b gene methylation were analyzed by MSP (D) Representative gel images of three biological replicates are presented. Data are presented as the mean $\pm \mathrm{SD}$ of three independent replicates. ${ }^{* *} \mathrm{P}<0.01$. circ, circular RNA; miR, microRNA; AML, acute myelogenous leukemia; MSP methylation-specific PCR; USP, unmethylation-specific PCR.

promotes the proliferation of AML cells through miR-34b. The Circ-ATAD1-miR-34b cell signaling axis, which contributed the tumor cell proliferation, has been revealed. However, methylation-related mechanisms were the major regulation mechanism between Circ-ATAD1 and miR-34b.

\section{Discussion}

BMMNCs are located in hematopoietic niches and play important roles in protecting leukemic cells from conventional chemotherapy (19). Leukemia initiating cells (LICs) interact with their surrounding bone marrow microenvironment (22), and the complex cell network surrounding LICs determines their fate (23). Various cellular signaling pathways and metabolic mechanisms regulate the BMMNC microenvironment and contribute to AML development $(24,25)$. However, the detailed regulation mechanism for BMMNC malignant transformation has not been clearly defined.

The involvement of circ-ATAD1 in AML, and its potential crosstalk with miR-34b, a tumor suppressor in AML (26), were investigated in the present study. As such, circ-ATAD1 was found to be upregulated in AML, which downregulated
miR-34b expression through methylation, promoting AML cell proliferation.

A previous study characterized circ-ATAD1 as an oncogenic circRNA in gastric cancer (16), where it was reported to be upregulated. Furthermore, circ-ATAD1 was found to sponge miR-140-3p to upregulate YY1 expression. Additionally, YY1 activates the transcription of phosphorylated CTD interacting factor 1 to promote cancer cell proliferation, invasiveness and migration (16). Consistently, circ-ATAD1 was found to be upregulated in AML in the present study, with enhancing effects on cancer cell proliferation. Therefore, circ-ATAD1 is likely an oncogenic circRNA in AML. However, as miRNA sponges, most circRNAs are primarily localized in the cytoplasm (27); the present study revealed that circ-ATAD1 was only detected in the nucleus, and not in the cytoplasm. Therefore, we hypothesize that circ-ATAD1 may be expressed in different subcellular locations in different cell types, though further confirmative investigation is required.

miR-34b was recently confirmed to target heat shock factor protein 1 in AML, which suppressed cell survival (26). Consistently, the present study showed that miR-34b was downregulated in $\mathrm{AML}$, and that its overexpression suppressed cellular proliferation, further confirming the 

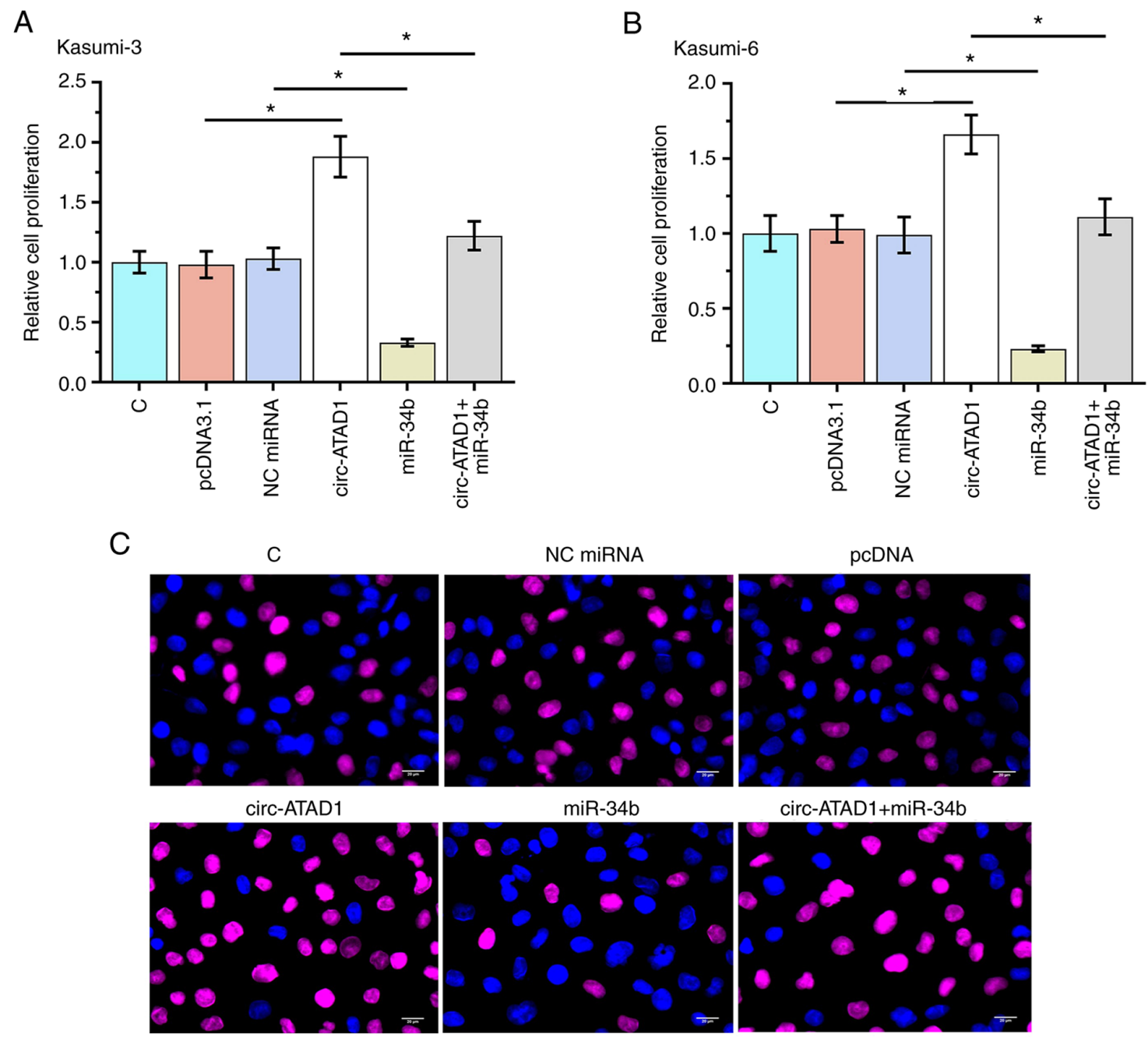

miR-34b

circ-ATAD1+miR-34b
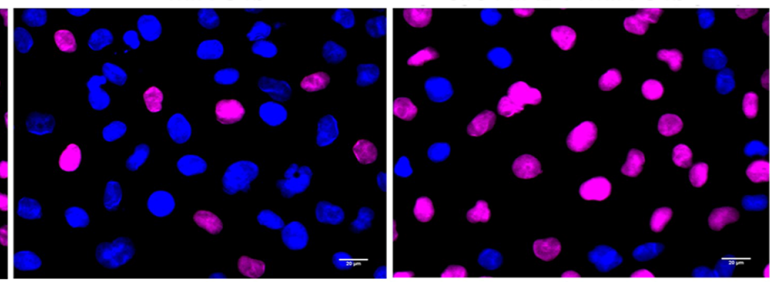

D

C
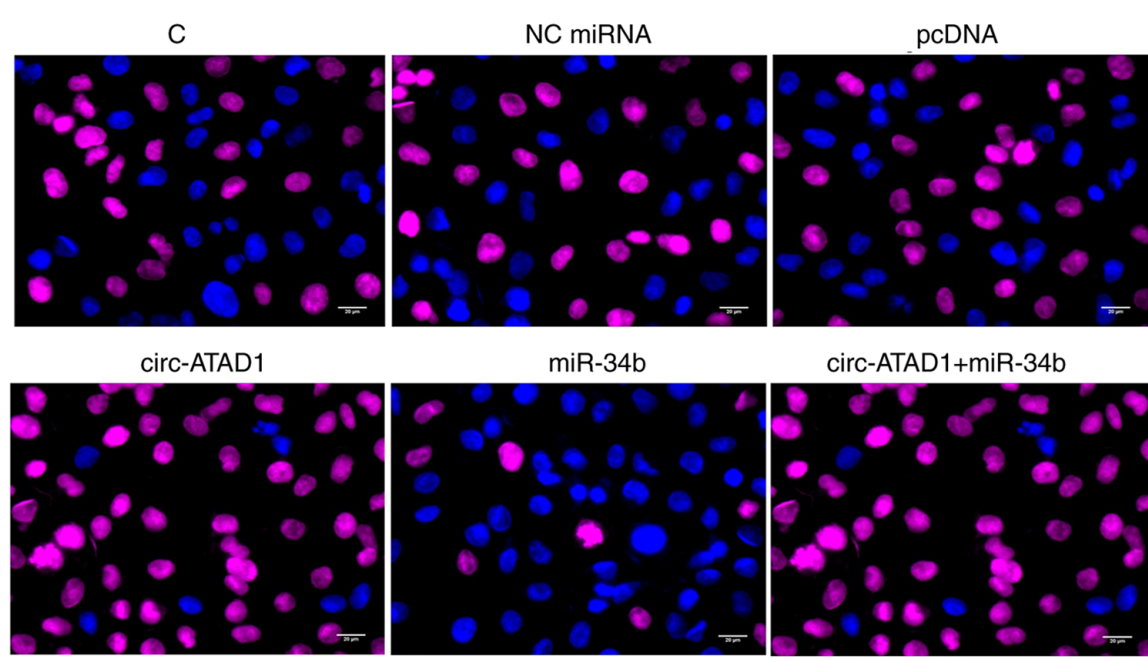

circ-ATAD1+miR-34b

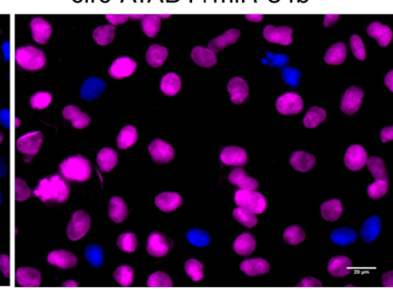

Figure 4. Circ-ATAD1 overexpression increases acute myelogenous leukemia cell proliferation through miR-34b. Role of circ-ATAD1 and miR-34b in regulating (A) Kasumi-3 and (B) Kasumi-6 cell proliferation was analyzed by BrdU assay. Representative images of (C) Kasumi-3 and (D) Kasumi-6 cells are displayed. Scale bar, $20 \mu \mathrm{m}$. Blue signals indicate DAPI staining; red signals indicate Brdu signaling Normalized data of three biological replicates are presented. Mean values of three replicates are presented. "P<0.05. circ, circular RNA; miR, microRNA; NC, negative control.

tumor suppressive role of miR-34b in AML. However, to the best of our knowledge, the upstream regulators of miR-34b in AML have not been reported previously. In the current study, circ-ATAD1 was shown to be a nucleus-specific circRNA. DNA methylation is known to occur in the nucleus (28), and numerous circRNAs potentially regulate the methylation of miRNAs in association with cancer biology $(29,30)$. Notably, the present study revealed that circ-ATAD1 downregulated miR-34b expression in AML by increasing methylation of the miR-34b gene promoter. Furthermore, miR-34b mimics 
reversed circ-ATAD1 overexpression-induced cellular proliferation, indicating an interaction between the two noncoding RNAs. However, a moderate correlation was observed between circ-ATAD1 and miR-34b across AML, but not the control samples. Therefore, certain AML-related factors may be involved in mediating the interaction between circ-ATAD1 and miR-34b. Collectively, the results of the present study revealed the circ-ATAD1-miR-34b axis as a novel regulatory signaling pathway specific to AML malignant transformation. However, its downstream genes, and the specific role and contribution in different cell types at different transformation phases, requires further investigation.

In conclusion, circ-ATAD1 is upregulated in AML and promotes AML cell proliferation by downregulating miR-34b via promoter methylation.

\section{Acknowledgements}

Not applicable.

\section{Funding}

This work was supported by the Natural Science Foundation of Inner Mongolia (grant no. 2019MS08047).

\section{Availability of data and materials}

The datasets used and/or analyzed during the current study are available from the corresponding author on reasonable request.

\section{Authors' contributions}

YZ put forward the concept, designed the experiments, provided general supervision, edited the manuscript and was a guarantor of integrity of the entire study. YW, BG and XQ acquired and analyzed the data. YW wrote the manuscript. $\mathrm{LB}, \mathrm{BL}$ and $\mathrm{HB}$ conducted the literature search and interpretated the data. XW and XYW defined intellectual content, conducted the literature search, were involved in interpreting the results and data analysis, and revised the manuscript. $\mathrm{YZ}$ and $\mathrm{YW}$ confirm the authenticity of all the raw data. All authors have read and approved the final manuscript.

\section{Ethics approval and consent to participate}

The present study was approved by the Ethics Committee of Xing'an League People's Hospital. All experiments were performed in accordance with the 1964 Declaration of Helsinki and its later amendments. Written informed consent to participate in the study was obtained from patients and controls prior to sample collection.

\section{Patient consent for publication}

Not applicable.

\section{Competing interests}

The authors declare that they have no competing interests.

\section{References}

1. Rose-Inman H and Kuehl D: Acute leukemia. Hematol Oncol Clin North Am 31: 1011-1028, 2017.

2. Parkin B, Ouillette P, Yildiz M, Saiya-Cork K, Shedden K and Malek SN: Integrated genomic profiling, therapy response, and survival in adult acute myelogenous leukemia. Clin Cancer Res 21: 2045-2056, 2015.

3. Schiller GJ: High-risk acute myelogenous leukemia: Treatment today and tomorrow. Hematology Am Soc Hematol Educ Program 2013: 201-208, 2013.

4. Gores GJ and Kaufmann SH: Selectively targeting Mcl-1 for the treatment of acute myelogenous leukemia and solid tumors. Genes Dev 26: 305-311, 2012.

5. Jiang XJ, Huang KK, Yang M, Qiao L, Wang Q, Ye JY, Zhou HS, Yi ZS, Wu FQ, Wang ZX, et al: Synergistic effect of panobinostat and bortezomib on chemoresistant acute myelogenous leukemia cells via AKT and NF-кB pathways. Cancer Lett 326: 135-142, 2012.

6. Piya S, Andreeff M and Borthakur G: Targeting autophagy to overcome chemoresistance in acute myleogenous leukemia. Autophagy 13: 214-215, 2017

7. Gill H, Leung AY and Kwong YL: Molecularly targeted therapy in acute myeloid leukemia. Future Oncol 12: 827-838, 2016.

8. Hatzimichael E, Georgiou G, Benetatos L and Briasoulis E: Gene mutations and molecularly targeted therapies in acute myeloid leukemia. Am J Blood Res 3: 29-51, 2013.

9. Konig $\mathrm{H}$ and Levis $\mathrm{M}$ : Is targeted therapy feasible in acute myelogenous leukemia? Curr Hematol Malig Rep 9: 118-127, 2014.

10. Martelli AM, Evangelisti C, Chiarini F and McCubrey JA: The phosphatidylinositol 3-kinase/Akt/mTOR signaling network as a therapeutic target in acute myelogenous leukemia patients. Oncotarget 1: 89-103, 2010.

11. Pei S, Minhajuddin M, Callahan KP, Balys M, Ashton JM, Neering SJ, Lagadinou ED, Corbett C, Ye H, Liesveld JL, et al: Targeting aberrant glutathione metabolism to eradicate human acute myelogenous leukemia cells. J Biol Chem 288: 33542-33558, 2013.

12. Vo JN, Cieslik M, Zhang Y, Shukla S, Xiao L, Zhang Y, Wu YM, Dhanasekaran SM, Engelke CG, Cao X, et al: The landscape of circular RNA in cancer. Cell 176: 869-881.e13, 2019.

13. Fang Y and Fullwood MJ: Roles, functions, and mechanisms of long non-coding RNAs in cancer. Genomics Proteomics Bioinformatics 14: 42-54, 2016.

14. Rücker FG, Russ AC, Cocciardi S, Kett H, Schlenk RF, Botzenhardt U, Langer C, Krauter J, Fröhling S, Schlegelberger B, et al: Altered miRNA and gene expression in acute myeloid leukemia with complex karyotype identify networks of prognostic relevance. Leukemia 27: 353-361, 2013.

15. Benassi B, Marani M, Loda M and Blandino G: USP2a alters chemotherapeutic response by modulating redox. Cell Death Dis 4: e812, 2013.

16. Zhang L, Chang X, Zhai T, Yu J, Wang W, Du A and Liu N: A novel circular RNA, circ-ATAD1, contributes to gastric cancer cell progression by targeting miR-140-3p/YY1/PCIF1 signaling axis. Biochem Biophys Res Commun 525: 841-849, 2020.

17. Wang J, Liu X, Lu H, Jiang C, Cui X, Yu L, Fu X, Li Q and Wang J: CXCR4(+)CD45(-) BMMNC subpopulation is superior to unfractionated BMMNCs for protection after ischemic stroke in mice. Brain Behav Immun 45: 98-108, 2015.

18. Rao X, Huang X, Zhou Z and Lin X: An improvement of the 2(-delta delta CT) method for quantitative real-time polymerase chain reaction data analysis. Biostat Bioinforma Biomath 3: 71-85, 2013.

19. Pavani RS and Elias MC: Following trypanosoma cruzi RPA-DNA interaction using fluorescent in situ hybridization coupled with immunofluorescence (FISH/IF). Methods Mol Biol 2281: 209-215, 2021.

20. Yuan Y, Wang Q, Ma SL, Xu LQ, Liu MY, Han B, Du N, Sun XL, Yin XL and Cao FF: 1ncRNA PCAT-1 interacting with FZD6 contributes to the malignancy of acute myeloid leukemia cells through activating Wnt/ $\beta$-catenin signaling pathway. Am J Transl Res 11: 7104-7114, 2019.

21. Mehdipour M, Etienne J, Chen CC, Gathwala R, Rehman M, Kato C, Liu C, Liu Y, Zuo Y, Conboy MJ and Conboy IM: Rejuvenation of brain, liver and muscle by simultaneous pharmacological modulation of two signaling determinants, that change in opposite directions with age. Aging (Albany NY) 11: 5628-5645, 2019. 
22. Ishikawa F, Yoshida S, Saito Y, Hijikata A, Kitamura H, Tanaka S, Nakamura R, Tanaka T, Tomiyama H, Saito N, et al: Chemotherapy-resistant human AML stem cells home to and engraft within the bone-marrow endosteal region. Nat Biotechnol 25: 1315-1321, 2007.

23. Nakamura-Ishizu A, Takubo K, Kobayashi H, Suzuki-Inoue K and Suda T: CLEC-2 in megakaryocytes is critical for maintenance of hematopoietic stem cells in the bone marrow. J Exp Med 212: 2133-2146, 2015.

24. Stevens AM, Xiang M, Heppler LN, Tošić I, Jiang K, Munoz JO, Gaikwad AS, Horton TM, Long X, Narayanan P, et al: Atovaquone is active against AML by upregulating the integrated stress pathway and suppressing oxidative phosphorylation. Blood Adv 3: 4215-4227, 2019.

25. Vignon C, Debeissat C, Bourgeais J, Gallay N, Kouzi F Anginot A, Picou F, Guardiola P, Ducrocq E, Foucault A, et al: Involvement of GPx-3 in the reciprocal control of redox metabolism in the leukemic niche. Int J Mol Sci 21: 8584, 2020.

26. Li G, Song Y, Zhang Y, Wang H and Xie J: MiR-34b Targets HSF1 to suppress cell survival in acute myeloid leukemia. Oncol Res 24: 109-116, 2016.
27. Hansen TB, Jensen TI, Clausen BH, Bramsen JB, Finsen B, Damgaard CK and Kjems J: Natural RNA circles function as efficient microRNA sponges. Nature 495: 384-388, 2013.

28. Massart R, Barnea R, Dikshtein Y, Suderman M, Meir O, Hallett M, Kennedy P, Nestler EJ, Szyf M and Yadid G: Role of DNA methylation in the nucleus accumbens in incubation of cocaine craving. J Neurosci 35: 8042-8058, 2015.

29. Liu Z, Yu Y, Huang Z, Kong Y, Hu X, Xiao W, Quan J and Fan X: CircRNA-5692 inhibits the progression of hepatocellular carcinoma by sponging miR-328-5p to enhance DAB2IP expression. Cell Death Dis 10: 900, 2019.

30. Gu Y, Ci C, Zhang X, Su M, Lv W, Chen C, Liu H, Zhang D, Zhang $\mathrm{S}$ and Zhang Y: Prediction of circRNAs based on the DNA methylation-mediated feature sponge function in breast cancer. Front Bioeng Biotechnol 7: 365, 2019.

(i) () () This work is licensed under a Creative Commons Attribution-NonCommercial-NoDerivatives 4.0 International (CC BY-NC-ND 4.0) License. 